J. Sustain. Wireless Syst.

Vol.01/ No. 01

Pages: 1-19

http://irojournals.com/irosws/

DOI: https://doi.org/10.36548/jsws.2019.1.001

\title{
THE EVALUATION OF PERFORMANCE FOR A MASS-MIMO SYSTEM WITH THE STSK SCHEME OVER 3-D $\alpha-\lambda-\mu$ FADING CHANNEL
}

\author{
Joy Iong-Zong Chen, \\ Professor, \\ Dept. Electrical Engineering of Da-Yeh University, Taiwan, ROC. \\ Email: jchen@mail.dyu.edu.tw
}

\begin{abstract}
The evaluation to the performance of bit error rate (BER) for an massive multi-input multi-output (MMIMO) system with spatial time shift keying (STSK) scheme over three-dimensions (3-D) $\alpha$ - $\lambda$ - $\mu$ fading model is proposed in this article. The long-term evaluation advanced (LTE-A) or 5th generation (5G) M-MIMO radio system is deployed with beamforming techniques over 3-D environment that contains the correlation phenomenon between angle of arrival (AoA) and angle of departure (AoD). Moreover, the determination of moment generating function (MGF) and characteristic function (CF) approaches is applied to derive analytical expression of BER for 3-D M-MIMO in order to avoid some difficult steps. Besides, the channel capacity is presented by using of mutual information to confirm the accuracy of the theoretically derived formulas. Furthermore, the work of performance evaluation is with different numbers of transmitter and receiver antennas. The correlation parameter between AoA and AoD definitely generates impact on the performance of BER when consider an M-MIMO system over the $\alpha-\lambda-\mu$ fading model and beamforming transmission.
\end{abstract}

Keywords: $\alpha-\lambda-\mu$ fading model, AoA, AoD, M-MIMO Beamforming, STSK.

\section{INTRODUCTION}

Recently, it is known that the infrastructure of the $5^{\text {th }}$ generation $(5 \mathrm{G})$ wireless systems is assigned with a structure of 5 layers, in which the Physical and Medium Access Control (MAC) layers are definitely impacting the system performance of a $5 \mathrm{G}$ cellular system. Especially, in wireless radio systems, overall system performance is highly affected by the statistical modeling and characterization of the wireless channel between the transmitter and the receiver sides. Therefore, the requirement of accurate modeling and efficiently design for the three-dimension (3-D) channel is necessary. It is also great important to estimate the available knowledge previously on certain parameters of the communication channel, such as, angle of departure (AoD) and angle of arrival (AoA), path loss, and 
J. Sustain. Wireless Syst.

Vol.01/ No. 01

Pages: 1-19

http://irojournals.com/irosws/

DOI: https://doi.org/10.36548/jsws.2019.1.001

multipath time delay spread measurements, when one models the channel consistent with the information available and held a joint probability distribution for the attribution of the channel matrix. The contribution of [1] provides measurements and channel models that may be used to design future $5 \mathrm{G}$ millimetre-waves (MMW) cellular networks and gives insight into antenna beam steering algorithms for these systems. In addition, the work in [2] described that the potential capacity gain is highly dependent on the multipath richness, since a fully correlated multi-input multi-output (MIMO) radio channel only offers one subchannel, while a completely decorrelated radio channel potentially offers multiple subchannels depending on the antenna configuration. So far, there are a huge number of researches explored the issue addressing in MIMO wireless radios. More recently, both an information theoretic channel for modeling a 3-D M-MIMO system and the performance evaluation by characterizing the prediction analysis to these systems is investigated in [3]. Authors work to maximize the energy efficiency by adjusting the training duration, training power, and data power under the constraint of the total transmit energy and spectral efficiency requirements. The scenario considered that users worked in the downlink M-MIMO frequency-division duplexing (FDD) system over a correlated Rayleigh fading channel [4]. Besides, the effort in deriving a generalized expression of the spatial correlation function (SCF) in a 3-D cellular layout, which compares with the results in performance for the FD-MIMO system over the uniform linear array (ULA) and the uniform circular array (UCA) configurations, is illustrated in [5]. There is a referred block-partition compressive sampling matching pursuit (BPCoSaMP) algorithm used to exploit the block-structure sparsity in angular domain proposed in [6], which claimed that is able to further improve channel estimation performance. The report in [7] summarizes the deployed scenarios and evaluation methodologies of the newly developed 3-D channel models, including antenna, path loss and fast fading modeling. The recent development of the full dimension MIMO (FD-MIMO) study in 3GPP is also discussed. A novel theoretical non-stationary 3-D wideband twin-cluster channel model for M-MIMO communication systems with carrier frequencies on the order of gigahertz $(\mathrm{GHz})$ is presented in [8]. Based on well-known propagation models and evaluation methods, such as average inter-site distances and traffic load, a novel method for performance evaluation of radio access network approaches is proposed in [9]. The method is allowing higher flexibility while maintaining reasonable complexity in which takes into account not only conventional dimensioning parameters, but also 3-D spatial user and traffic distribution. In [10] authors investigate the low-complexity channel reconstruction methods for downlink precoding in an M-MIMO systems. In that the user is allocated much less streams than the number of its antennas, the base station 
J. Sustain. Wireless Syst.

Vol.01/ No. 01

Pages: 1-19

http://irojournals.com/irosws/

DOI: https://doi.org/10.36548/jsws.2019.1.001

(BS) utilizes the singular value decomposition (SVD) factorizations to get the effective channels in time division duplex (TDD) mode. The other study in [11] aims the performance evaluation of that can be deployed as a temporary micro cell in dense-population areas. In the work proposes lower-height HAP system, the service antenna array is still much higher than the users, hence it is likely to form a strong line-of-sight (LoS) channel. Besides, an extensive channel sounding campaign in an urban macro cell using circular antenna arrays on both link ends is described in [12]. The statistical analysis results are also discussed based on the high resolution parameter estimation framework of a maximum likelihood framework for parameter estimation (RIMAX). Except the previously mentioned results, there are also other research reports of the evaluation to the MIMO-beamforming signaling obtained from the assumption with both azimuth and elevation directions for wave propagation channel, i.e., include the factor of 3-D channel to discuss the analyzed performance of a communication radio with the MIMO [13]. Laterly, an efficient dispersion matrix optimization technique for the recent concept of Space Time Shift Keying (STSK) has been introduced [14]. The scheme of STSK exploits to the STSK-specific signal structure relying on the dispersion-matrix activation instead of the classic spatial multiplexing. The computational complexity of STSK imposed by the system optimization is reduced by tens of times when compared to that of the general approach. Based on the advantages of STSK that provides an efficient trade off between diversity and multiplexing without inter channel interference and without the need for large antenna arrays, authors in [15] introduces a viable MIMO solution for high bit-rate transmission in the E-band. The most significant channel impairments related to small-cell backhaul in dense urban environment is also considered in the investigation.

In the previous work, the authors' work was only to express the current $3 \mathrm{GPP}\left(3^{\text {rd }}\right.$ generation partnership project) activity around simplified three-dimension beamforming (3-D-BF) and FD-MIMO. They have provided the basic concept to enhance the understanding of the current industrial challenges as well as to deeply hint the standards' vision for 3-D-BF. Authors took the specifications both azimuth and horizon fading channels of one subscribe into consideration for the interference coordination to maximize both the cell-edge user's and cell center users' throughput in [16]. In [17] an extension of the International Telcommunications Union 2-dimision (ITU2D) channel model to 3-D was proposed, in which was with adding a distance dependent elevation spread based on observations from ray tracing. Through system-level simulations which came out from the aforementioned literatures, authors observe that the behavior of M-MIMO is greatly impacted by the modeling of the 3-D channel. Now a day a popular 3-D fading channel for M-MIMO beamforming proposed is so-called $\alpha-\lambda$ - $\mu$ fading channel, 
J. Sustain. Wireless Syst.

Vol.01/ No. 01

Pages: $1-19$

http://irojournals.com/irosws/

DOI: https://doi.org/10.36548/jsws.2019.1.001

which is a general small-scale fading model for wireless digital communications, and explores the nonlinearity and at the same time the inhomogeneous nature of the propagation medium [18]. In [19], researchers analyzed and evaluated the performance of digital communication systems that operate over the $\alpha-\lambda-\mu$ fading channels. The correctness of the derived formulas results for the aforementioned work was numerically compared with Monte Carlo simulation results. Both results are in perfect agreement over a wide range of average signal-to-noise ratio (SNR) and different values of the fading parameters.

This paper focuses on performance evaluation of BER and outage probability for an M-MIMO system over correlated 3-D $\alpha-\lambda-\mu$ channel model and beamforming technique. The assumption of fading model involves spatial correlation effect in the transmitting and receiving branches. Moreover, authors also considered that the deployment involves an arbitrary correlation coefficient and the AoA parameter. Furthermore, both transmitter and receiver environment is assigned with different antenna numbers, and the receiver is considered to combine with maximal ratio combining (MRC) for a 3-D M-MIMO system operating over correlated $\alpha-\lambda-\mu$ fading channel.

This paper is organized as follows. In Section II, the 3-D spatial channel model for M-MIMO system and both the modulation and de-modulation schemes are demonstrated first. In Section III, the performance evaluation of channel capacity and BER for a 3-D M-MIMO system with $\alpha-\lambda$ - $\mu$ fading scenarios are presented. A validation of the derived theoretical formulas with numerical manifestation is illustrated in Section IV. Finally, Section V concludes the report.

\section{SYSTEM MODEL DESCRIPTIONS}

The description to the deployment of the system model for M-MIMO scheme and the relevant environments is necessary, before the exploration to the performance of M-MIMO beamforming with STSK modulation over 3-D channel model is studied.

\subsection{The Adopted Spatial Channel Model}

Based on the mobile communication radio standards, a spatial channel model for MIMO simulations is proposed shown in Fig. 1 [17], in which through statistical parameters, such as amplitude, AoA, AoD, and path delay are described. The original system architecture has been proposed in [17], in which the channel of a 3-D M-MIMO system was proposed by the same researchers. However, they were not refer the beamforming schemes. To overcome the drawback, the investigation of applying the use of a 3-D M- 
MIMO system with beamforming technique in this article and assuming that antennas are arranged for a 3-D array where each column contains $M$ antenna elements. A simple deployment of a 3-D M-MIMO channel model is shown in Fig. 1 in which the scenario can be described according to the contents of 5G network. For instance, there are four local areas circled with red dash color to express they containing different environments, such as femtocell to femtocell for living room, femtocell to lap-top for different floor, and some show other scenario graphically. On the other hand, the circled with red color can be seen as the beam subset distributed by the Macrocell BS where has the M-MIMO signaling scheme. Furthermore, the 3-D channel model puts on an azimuth plane considered in this investigation is demonstrated at the right up corner of Fig. 1.

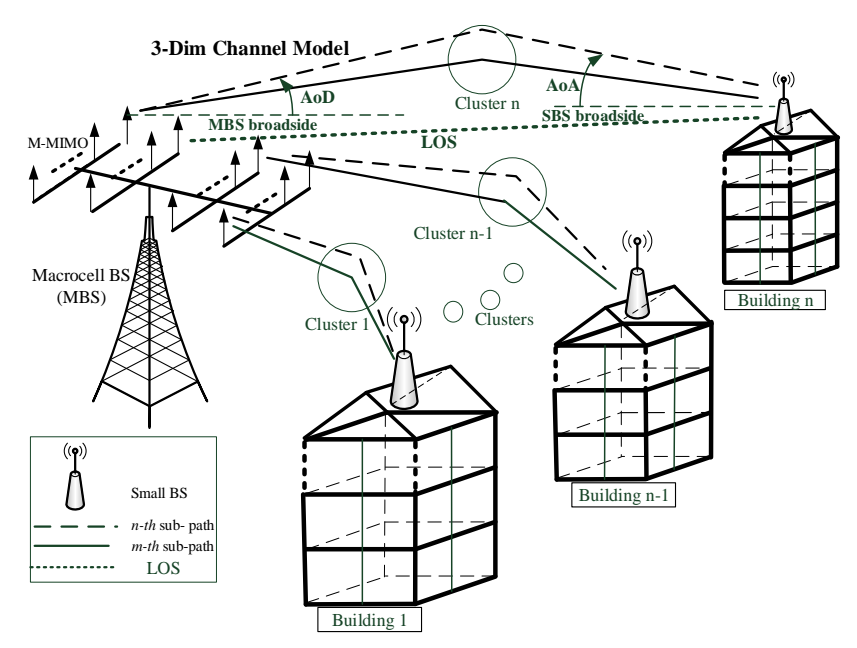

Fig. 1. The Basic Infrastructure of a 3-D M-MIMO with Beamforming for 5G Radio System.

There are exactly $K$ antenna elements per antenna port with a pattern $A_{E}$ is given by

$$
A_{E}\left(\phi_{e}, \theta\right)=\mathrm{G}_{E, \text { max }}\left\{-\left(A_{H}\left(\phi_{e}\right)+\mathrm{A}_{V}(\theta)\right), \mathrm{A}_{m}\right\}
$$

where $A_{m}=30 d B, G_{E, \max }=8 d B i$ and $A_{H}\left(\phi_{e}\right)=-\min \left[12\left(\phi_{e} / \phi_{3 d B}\right)^{2}, A_{m}\right]$, where $\phi_{3 d B}=65^{0}$, moreover, where $A_{V}(\theta)=-\min \left[12\left(\theta-\theta_{\text {titt }} / \theta_{3 d B}\right)^{2}, \mathrm{SLAV}\right]$, in which $\mathrm{SLAV}=30$ and $\theta_{3 d B}=65^{\circ}$. A simple deployment of 3D channel model is shown in Fig. 1 which is discussed in [13]. In addition, there is a 3-D channel model works within the azimuth plane and constructs to play as the infrastructure for a wireless communication system, in which the BS (base station) is embedded with MIMO (remark: here MIMO is considered as multi-antenna) signaling scheme. Since the space is limited, the description to the configuration of antenna ports is ignored. There are two situations, either choosing the number of antenna ports per column is assumed $M$ and $K=M$ or setting $K=1$ are considered for the 3GPP group Technique 
Specification Group-Radio Access Network Working Group 1 (TSG-RANWG1). A weighted sum of channels with the $K$ elements is assigned in the element. The channel port is given by

$$
\left[h_{u, s, n}(t ; \tau)\right]=\sum_{\text {antenna } e \text { port s }} \omega_{\text {antenna }}\left[h_{u, n}(t ; \tau)\right]_{\text {antenna u }}
$$

where the summation presented above is performed over all antenna elements in port $s$. Accordingly, if the real 3-D channel is considered, then each antenna port adopts as $s$ and the mobile station (MS) antenna port $u$ is deployed in [3], and which is rewritten as

$$
\begin{aligned}
& h_{u, s, n}(t ; \tau)=\sum_{m=1}^{M}\left[\begin{array}{c}
{ }^{F} R x, u, V\left(\varphi_{n, m}\right) \\
{ }^{F} R x, u, H\left(\varphi_{n, m}\right)
\end{array}\right]\left[\begin{array}{ll}
\alpha_{n, m}^{V V} & \alpha_{n, m}^{V H} \\
\alpha_{n, m}^{H V} & \alpha_{n, m}^{H H}
\end{array}\right]\left[\begin{array}{c}
{ }^{F} T x, u, V\left(\phi_{n, m}\right) \\
{ }^{F} T x, u, H\left(\phi_{n, m}\right)
\end{array}\right] \\
& \quad \times \exp \left(j 2 \pi \lambda_{0}^{-1}\left(\bar{\phi}_{n, m} \cdot{ }^{\bar{F}} R x, u\right)\right) \exp \left(j 2 \pi \lambda_{0}^{-1}\left(\bar{\phi}_{n, m} \cdot{ }^{\bar{F}} T x, s\right)\right) \times \exp \left(j 2 \pi v_{n, m} t\right) \delta\left(\tau-\tau_{n, m}\right)
\end{aligned}
$$

where $\alpha_{n}$ indicates the complex random amplitude of the $n$-th path, then $\sqrt{g_{t}\left(\phi_{n}, \theta_{n}, \theta_{i t i}\right)}$ and $\sqrt{g_{r}\left(\varphi_{n}, \vartheta_{n}\right)}$ represent the global pattern of transmitter and the receiver antenna, respective. The $d_{t}$ and $d_{r}$ are the separation between transmitter and receiver antenna port that considered as $2 \pi / \lambda$, where $\lambda$ is the wave length of the carrier frequency, and $\phi_{n}$ and $\theta_{n}$ denote the azimuth and elevation AoD of the $n$-th path, respectively, $\varphi_{n}$ and $\vartheta_{n}$ are the azimuth and elevation angle of the antenna boresight. Consider MMIMO beamforming scheme employs $N_{T}$ transmitter and $N_{R}$ receiver antennas which is equipped with the receiver signal vector $Y_{R} \in \mathrm{C}^{N_{R} \times 1}$ and the transmit signal vector $X_{T} \in \mathrm{C}^{N_{T} \times 1}$, respectively. One more variable, $\theta_{\text {tilt }}$ is the elevation angle of the antenna boresight. Normally, the radio M-MIMO channel is an $N_{T} \times N_{R}$ matrix and which is able to be re-written as

$$
\begin{aligned}
h(t, \tau)= & \iint{\sqrt{g_{r}\left(\varphi_{n}, \vartheta_{n}\right)}}^{T} h(t, \tau, \Omega, \Psi) \sqrt{g_{t}\left(\phi_{n}, \theta_{n}, \theta_{\text {tilt }}\right)} \\
& \times \overrightarrow{\mathrm{a}}_{R}(\Psi)\left(\overrightarrow{\mathrm{a}}_{T}(\Omega)\right)^{T} d \Omega d \Psi
\end{aligned}
$$

where $\sqrt{g_{r}\left(\varphi_{n}, \vartheta_{n}\right)}$ and $\sqrt{g_{t}\left(\phi_{n}, \theta_{n}, \theta_{i t k}\right)}$ are the patterns of the receiving and transmitting antennas, respectively. $\sqrt{g_{r}\left(\varphi_{n}, \theta_{n}\right)}$ and $\sqrt{g_{t}\left(\phi_{n}, \theta_{n}, \theta_{t i t}\right)}$ are $2 \times 1$ vectors whose entries represent the vertical and horizontal field patterns, when polarization is considered. Vectors $\overrightarrow{\mathrm{a}}_{R}(\Psi)$ and $\overrightarrow{\mathrm{a}}_{T}(\Omega)$ are corresponding to the array responses of the transmitting and receiving antennas, and whose entries are given by $\left[\vec{a}_{R}(\Psi)\right]_{i}=\exp \left(j \overrightarrow{\mathrm{k}}_{R, \Psi} \overrightarrow{\mathrm{x}}_{R . i}\right),\left[\vec{a}_{R}(\Psi)\right]_{i}=\exp \left(j \overrightarrow{\mathrm{k}}_{\mathrm{T}, \Psi} \overrightarrow{\mathrm{x}}_{\mathrm{T} . i}\right)$, respectively, and where $\overrightarrow{\mathrm{x}}_{R . i}$ is the location vector of the $i-$ th receiving antenna, whereas $\overrightarrow{\mathrm{x}}_{\mathrm{T} . i}$ is that of the $i$-th transmitting antenna. The transmitted signal power is constricted as the value equivalent to the number of transmitter antennas, i.e., $P_{T}=E\left[\left\|X_{R}\right\|^{2}\right]=N_{T}$, and the MIMO channel complex matrix $h \in C^{N_{T} \times N_{R}}$ is characterized as the spatially corrected distributed. The nocoherent $\mathbf{Z} \in \boldsymbol{C}^{N_{R} \times N_{T}}$ channel matrix can be written as $\bar{h}=M_{R}^{1 / 2} Z M_{S}^{1 / 2}$, where is including i.i.d Gaussian entries with zero mean and unit variance. On the other hand, $\mathbf{Z} \sim C N_{M_{R} M_{S}}\left(0, \sigma_{M_{R} M_{S}}\right)$, where $\sigma_{M_{R} M_{S}}$ is the covariance matrix of the random matrix $\mathbf{Z}$, and $\mathbf{M}_{R}$ and $\mathbf{M}_{S}$ are corresponding to denote the matrices of 
the receiving and transmitting spatial correlation. In addition, the $n$-th eigenvalue of $\mathbf{M}_{R}$ and $\mathbf{M}_{S}$ are designated as $\lambda_{i}^{M_{r}}, i=0,1, \cdots, N_{R}-1$ and $\lambda_{i}^{M_{s}}, i=0,1, \cdots, N_{T}-1$, respectively. It is known that the spatial correlation matrices can be decomposed by using of eigenvalue method in advanced, and re-written as $M_{R}=U_{M_{R}} \Lambda_{M_{R}} U_{M_{R}}^{\dagger}$ and $M_{S}=U_{M_{S}} \Lambda_{M_{S}} U_{M_{S}}^{\dagger}$, respectively. Furthermore, the symbol ${ }^{\dagger}$ in last equations denotes conjugate transpose, and $U_{M_{R}}$ and $U_{M_{S}}$ are containing the corresponding entries of the eigenvectors with respect to the related eigenvalues, while $\Lambda_{M_{R}}$ is a diagonal matrix with eigenvalues which are obtained from spatial correlation matrices in the main diagonal elements.

\subsection{Theoretical Review to the Coherent STSK Modulation Scheme}

The concept of STSK modulation is likely to consider a modulated signal $s(i)$ mixed with a pre-assigned dispersion matrix in advance of transmission and shown in Fig. 2, in which the set of $n$ bits forming the transmitted block are taken from the interleaved output of a traditional encoder at rate $\mathrm{k} / \mathrm{n}$. On the other hand, the modulation result of the transmitted complex symbol, $S_{g}(i)$, is given as

$$
S_{g}(i)=s(i) \cdot D_{g}(i)
$$

where the modulated signal $s(i)$ is considered as the length of $\log _{2}(i)$ number of input bits, and with the complex-valued symbol of the traditional modulation scheme, such as $M$-ary QAM (quadrature amplitude modulation) or $M$-ary PSK (phase-shift keying), and there are $G$ space-time dispersion matrices $D_{g}(i) \in C^{R * T}$ which can be written as

$$
D_{g}(i)=\left[\begin{array}{ccc}
d_{1}^{1} & \cdots & d_{1}^{T} \\
\vdots & \ddots & \vdots \\
d_{N}^{1} & \cdots & d_{N}^{T}
\end{array}\right], g=1, \cdots, G
$$

where only one dispersion matrix is enabled during each symbol propagation, and all zero matrices are disabled. Then the $R$ antenna array are going in charge of coordinating the transmission duty for every column of the selected dispersion matrix. Specifically, consider that the input data bits of STSK, $B$, are S/P (serial-to-parallel) combined with $b_{A}=\log _{2}(G)$ bits and $b_{B}=\log _{2}(M)$ bits. Thus, one generated the $M$ dispersion matrices $D_{g}$ shown in (5) is activated according to the $b_{A}$ bits, and the $b_{B}$ bits are in terms of the QAM symbols. Eventually, the source bits with a total length of $\log _{2}(M \cdot G)$ are mapped to each space-time block $S_{g}(i) \in C^{R \times T}$, where $R$ and $T$ represent the number of transmit antenna and the number of symbols per space-time block, respectively. The codeword of space-time is then arose from the equation shown in (5).

Accordingly, an extra method of transmitting further message bits for the STSK is generated in advance. The number of input bits, $\log _{2}(M)$, will determine the specified matrix $D(i)$ selected from which of the $G$ 
space-time dispersion matrices $D_{g}, g=1, \cdots, G$. Consider the situation that a 3 source bits per space-time block, there are $M$ size of the constellations and several possible combinations of the number of dispersion matrices $D_{g}$ set. Specifically, suppose that there is a fixed number of $\left.\log _{2} G \times M\right)$ bits per space-time block $S_{g}(i)$ are transmitted by applying M-ary QAM scheme, and for the special case of $(G \times M)=(1,8 ; 2,4 ; 4,2 ; 8,1)$.

Totally, there are four parameters involved in the STSK transmission, they are including the cardinality of the set of dispersion matrices $R$ and $T$, the number of the transmit antennas $N_{T}$, the number of the receive antenna $N_{R}$. It is assumed that $N_{T}=N_{R}$ in a backhaul system [14].

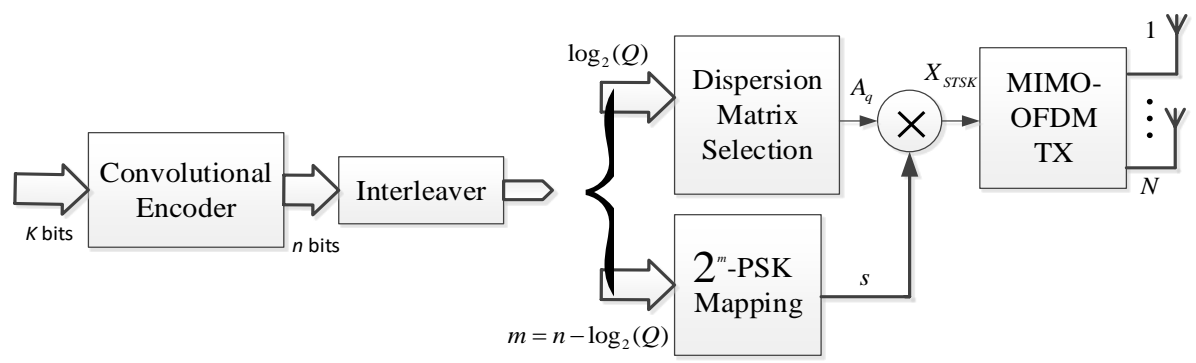

Fig. 2 The Block Diagram of STSK Modulation Scheme

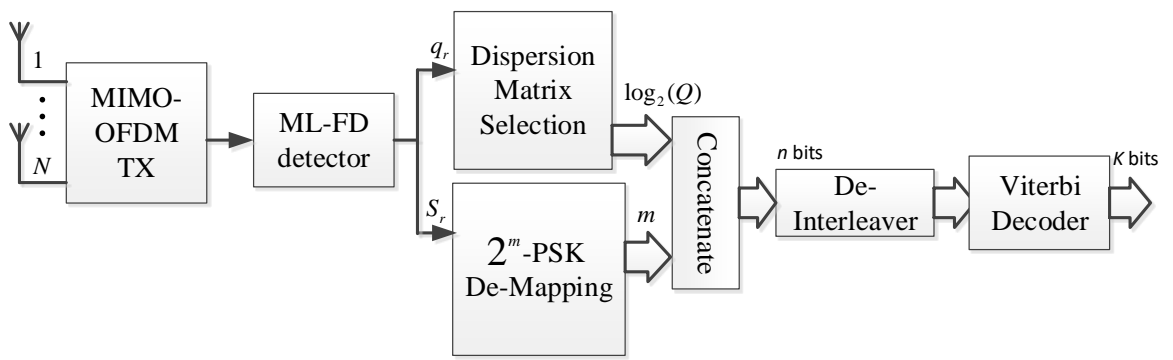

Fig. 3 The Block Diagram of STSK De-modulation Scheme

Once the propagating channel scenario is constructed completed, the received signal intensity, $\mathbf{Y}_{R} \in C^{N_{T} \times 1}$, for each spatial correlated channel experienced fading environment can be expressed as

$$
\mathbf{Y}_{R}=\sqrt{E_{s} / N_{T}} h X_{T}+N
$$

where $E_{S}$ is the symbol energy, $\boldsymbol{X}_{T} \in C^{N_{T} \times 1}, N \in C^{N_{R} \times 1}$ denote the transmit signal energy which is constrained in $\left[\left|\mathbf{X}_{T} \mathbf{X}_{T}^{\dagger}\right|^{2}\right]=N_{T} \mathbf{I}_{N_{R}}$, and the additive Gaussian noise vector with zero-mean and covariance $E\left[\mathbf{N} \mathbf{N}^{\dagger}\right]=N_{0} \mathbf{I}_{N_{R}}$ respectively, where $N_{0} / 2$ denotes as double-sided power spectral density of additive Gaussian White noise (AWGN). The signal-to-noise ratio (SNR) at the receiver is defined as $\gamma_{s}=E_{s} / N_{0}$. As the block diagram of convolutionally-encoded STSK demodulation shown in Fig. 3, in which the corresponding receiver scheme demodulates all the total $\log _{2}(Q)$ bits selecting the dispersion matrix and the remaining $m$ 
bits indexing the $2^{m}$-ary PSK symbol encoded STSK matrix columns transmitted over $P$ OFDM subcarriers. It is known that the correlated fading points happen over the fading channel determine the relative performance of the transmission schemes, and are shown to depend explicitly on the channel statistics through the eigenvalues of the spatial correlation matrices. It is the motivation to propose a practical implementation of the adaptive approach that switches between different MIMO signaling modes, with each mode comprising a particular combination of a MIMO scheme as well as modulation and coding scheme presented in this paper finally. This practical system is shown as follows to yield significant spectral efficiency improvements over non-adaptive transmission in typical channel scenarios.

\section{THE PERFORMANCE EVALUATION WITH MGF}

In the section the performance of bit error rate (BER) for a system with 3-D M-MIMO beamforming transmission is considered with the skill by looking for the moment generating function (MGF) first. Accordingly, it is known that the BER probability teaches the well calculation of the integral value which obtained by integrating the conditional pdf. The next step is applying the determination of BER for 5G systems operating over 3-D beamforming environment and it is characterized as

$$
P_{\mathrm{pdf}}^{3-D \mathrm{BF}}=\int_{-\infty}^{\infty} \int_{\phi_{L}}^{\phi_{U}} \int_{\theta_{L}}^{\theta_{U}} f\left(\gamma_{s} \mid \phi_{e}, \theta\right) \cdot f_{\theta_{n, m}, \phi_{n, m}}\left(\phi_{e}, \theta\right) d \gamma_{s} d \theta d \phi_{e}
$$

where $f_{\theta_{n, m}, \phi_{n, m}}\left(\phi_{e}, \theta\right)$ is the joint pdf of AoA and AoD which will be discussed later, $f\left(\gamma_{s} \mid \phi_{e}, \theta\right)$ represents the conditional pdf of $S N R$ between the input vector, $S$, and the output $y$ of the M-MIMO signaling scheme, $\theta_{L}$ and $\theta_{U}$ are corresponding to the minimum angle and maximum angle of AoA. There is the same case that is for the AoA, AoD has also the minimum and maximum angle values while the 3-D channel is considered. Thus, $\phi_{L}$ and $\phi_{U}$ indicate the minimum and maximum AoD, respectively. On the other hand, the minimum and maximum AoA depends on the maximum power ( $3 \mathrm{~dB}$ point) can be supplied from the 3-D M-MIMO system. Originally, the AoA and AoD come from the 3-D channel consideration which involved the azimuth, $\theta$, and the elevation, $\phi_{e}$, respectively [21].

Traditionally, the performance calculation of BER for a general wireless communication system employs the statistical operation of pdf and even tries to remove the condition terms eventually. However, in order to avoid the performance calculation of BER could meet some difficult steps, by means of determining the MGF is necessary generally. By applying the basic concept of random stochastic to determine the MGF of $S N R, \gamma_{s}$, and first then it can be naturally expressed as

$$
M g_{\gamma_{s}}(s)=E_{h}\left[\exp \left(-s \gamma_{s}(x)\right)\right]=\prod_{i=0}^{N_{R}} 1 /\left(1+s c x \lambda_{i}\right)
$$


where $E_{h}[\cdot]$ denotes the expectation operation with respect to $h$, which has been defined in (1). The MGF defined in last equation is for spatially correlated $\alpha-\lambda-\mu$ MIMO channel in which there are $n$ multipath clusters and a propagation environment as shown in Fig. 1. Accordingly, the $1^{\text {st }}$ Building to $n$ th Building have been assigned multi-path clusters number $\{1, \cdots, n-1, n\}$, respectively. The resulting signal envelope of the multipath is observed as a nonlinear function combined with the sum of the components come from the in-phase and quadrature components. The in-phase and quadrature components of the multipath waves of cluster $n$ are assigned as $X_{n}$ and $Y_{n}$, respectively. Then the resulting envelope in the form of a power parameter $\alpha>0$ can be expressed as

$$
R^{\alpha}=X^{2}+Y^{2}=\sum_{n=1}^{N} R_{n}^{\alpha}=\sum_{n=1}^{N} X_{n}+Y_{n}
$$

where $X_{n}$ and $Y_{n}$ are considered as zero-mean mutually correlated Gaussian processes, that is, $E\left[X_{n}\right]=E\left[Y_{n}\right]=0$ and with identical variances such that $E\left[X_{n}^{2}\right]=E\left[Y_{n}^{2}\right]=\hat{\gamma}^{\alpha} / 2 n$, where let's assign $\Omega=\hat{\gamma}^{\alpha}$. Now, if $Z=X$ or $Z=Y$ are assumed, then

$$
Z^{2}=\sum_{n=}^{N} Z_{i}^{2}
$$

Moreover, the in-phase and quadrature components of the fading signal within each clusters are correlated with correlation coefficient $\lambda$, which have zero-mean values, and identical powers. On the other hand, the correlation $\lambda$ as the name of the $\alpha-\lambda-\mu$ distribution is results of considered as inhomogeneity, and which can be calculated as $\lambda=2 n E\left[\mathrm{X}_{n} \mathrm{Y}_{n}\right] / \Omega$, where $0 \leq \lambda \leq 1$. Normally, the parameter $\mu$ as in the distribution name is related to the number of multipath clusters of the environment ever aforementioned. Generally, $\alpha>0$ is an arbitrary parameter for a fading signal with envelope $R$. Following the developing to the mentioned above for the multipath, the joint distribution $f_{X_{n} Y_{n}}\left(x_{n}, y_{n}\right)$ can be determined by the standard transformation procedure of variables in stochastic process, and the pdf (probability density function), $f_{R_{n}}\left(r_{n}\right)$, of $r_{n}$ shown in (a1) is able to be obtained as [18]

$$
f_{R_{n}}\left(r_{n}\right)=\frac{2 \sqrt{\pi} \mu^{\mu+0.5} \alpha r^{\alpha(\mu+0.5)-1}}{\Gamma(\mu)\left(\hat{r}^{\alpha}\right)^{\mu+0.5} \lambda^{\mu-0.5} \sqrt{1-\lambda^{2}}} \exp \left(-\frac{2 \mu r_{n}^{\alpha}}{\left(1-\lambda^{2}\right) \hat{r}^{\alpha}}\right) \mathbf{I}_{\mu-0.5}\left(\frac{2 \lambda \mu r_{i}^{\alpha}}{\left(1-\lambda^{2}\right) \hat{r}^{\alpha}}\right)
$$

where the $\alpha$-root mean value of the envelope $R_{n}$ is presented as $\hat{r}=\left(E\left[\mathrm{R}_{n}^{\alpha}\right]\right)^{1 / \alpha}, I_{\mu-0.5}(\cdot)$ is the modified Bessel function of the first kind and arbitrary order of real value, and $\Gamma(k)=\int_{0}^{\infty} t^{k-1} \exp (-t) d t$ denotes the Gamma function. The MGF of $\alpha-\lambda-\mu$ distribution is able to be determined by using of the definition of which can be calculated as [18] 


$$
\begin{aligned}
& M g_{\gamma}(s)=\frac{\alpha \sigma^{\mu} \mu^{\mu+0.5}}{\Gamma(\mu)(\hat{\gamma} s)^{0.5 \alpha(\mu+0.5)} \Sigma^{\mu-0.5}} \times \\
& \mathrm{H}_{1,0: 0,0,1,1,2,2}^{0,1: 1,1,1,1}\left[\begin{array}{cc}
\frac{2 \mu(\sigma-\Sigma)}{(\hat{\gamma} s)^{0.5 \alpha}} & (1-0.5 \alpha(\mu+0.5) ; 0.5 \alpha, 0.5 \alpha):-;(0.5,1) \\
\frac{4 \mu \Sigma}{(\hat{\gamma} s)^{0.5 \alpha}} & -:(0,1) ;(\mu-0.5,1),(0.5-\mu, 1)
\end{array}\right]
\end{aligned}
$$

where $H_{1,0: 0,1,1,2,2}^{0,1: 0,1,1}[\cdot]$ is the Fox's H-function, $\Sigma=\lambda / 1-\lambda^{2}$, and $\sigma=1 / 1-\lambda^{2}$. Consider the correlation between AoA and AoD corresponding to each multipath cluster are denoted as $\left\{\lambda_{1}, \lambda_{2}, \cdots, \lambda_{i}\right\}$. The eigenvalues are generally considered as $\lambda_{1} \geq \lambda_{2} \geq \cdots \geq \lambda_{i}>0$, and there exist $N$ distinct values in $\left\{\lambda_{i}\right\}$, where $i$ is defined as $i=\{1, \cdots, \max ($ number of $A o A$ or $A o D)\}$.

On the other hand, the AoA of the input/output signals is suitable for the issue addressed in the MMIMO system employs 3-D BF with MRC receiver, the covariance matrix, which now it is replaced with, $\boldsymbol{Q}_{\text {3-Dim-BF }}$, in the instantaneous mutual information could be implicitly decomposed as $\boldsymbol{Q}_{3 \text {-Dim-BF }}=U_{M_{S}} \Lambda_{3-\text { Dim-BF }} U_{M_{R}}$, with $\Lambda_{3-\operatorname{Dim}-\mathrm{BF}}=\operatorname{Diag}\left[E_{s}, 0, \cdots, 0\right]$. Hence, by substituting the channel matrix into mutual information formula $\Xi(S, \lambda)$, the new mutual information becomes

$$
\begin{aligned}
\Xi(S, \lambda)= & \log _{2}\left[\left(\boldsymbol{I}_{3-\mathrm{D} \mathrm{BF}}+\left(E_{s} / N_{0}\right) \times\right.\right. \\
& \left.\mathbf{M}_{R}^{1 / 2} \mathbf{Z M}_{S}^{1 / 2} \boldsymbol{Q}_{3-\mathrm{D} \mathrm{BF}}\left(\mathbf{M}_{S} \mathbf{Z M}_{R}\right)^{1 / 2}\right]
\end{aligned}
$$

where $\boldsymbol{I}_{3-\mathrm{D} \mathrm{BF}}$ is a unit matrix with same dimension of MRC, $E_{s} / N_{0}$ represents the $S N R, \mathbf{M}_{R}, \boldsymbol{Z}$, and $\mathbf{M}_{S}$ have been defined in last section. By adopting the results of covariance matrix $\boldsymbol{Q}_{3-\mathrm{D} \mathrm{BF}}$ and then combined them with taking the eigenvectors of matrices. The equation (14) can be re-written as $\Xi(S, \lambda)=\log _{2}\left[\boldsymbol{I}_{3-\mathrm{D} \mathrm{BF}}+\left|\lambda_{\max }^{M_{S}} \cdot \gamma_{s} \cdot \zeta \zeta^{\dagger}\right|\right]$, where $\gamma_{s}$ has been defined at last section, $\lambda_{\max }^{M_{S}}$ indicts the maximum value selected from the eigenvalues found in the matrix $\mathbf{M}_{s}$, i.e., $\lambda_{\max }^{\mathbf{M}_{s}}=\max \left(\lambda_{n}^{\mathbf{M}_{s}}\right), n=0,1, \cdots, N_{R}-1, \zeta$ is expressed as $\zeta=\left[\left(\lambda_{1}^{\mathbf{M}_{R}}\right)^{0.5} z_{1}, \cdots,\left(\lambda_{N_{R}}^{\mathbf{M}_{R}}\right)^{0.5} z_{N_{R}}\right]^{T}$, in which $z_{n}, n=0,1, \cdots, N_{R}-1$, represents the $n$-th entry of first column generated from matrix $Z$, and $z_{n} s^{\prime}$ denote independent, identically distributed (i.i.d.) zero-mean and unit variance complex Gaussian random variables. By using some simple operations of linear algebra the equation of $\left|\mathbf{I}_{u}+\mathbf{X Y}\right|=\left|\mathbf{I}_{v}+\mathbf{Y X X}\right|$ in which $\mathbf{X} \in C^{u \times v}$ and $\mathbf{Y} \in C^{v \times u}$ is able to be solved completed. Substitute the previously mentioned equation back into (14), and which becomes as $\Xi(S, y)=\log _{2}(1+\eta \Omega)$, where $\eta=\sum_{n=0}^{N_{R}-1} \lambda_{n}^{\mathbf{M}_{R}} \cdot \varepsilon_{n}, \Omega=\gamma_{S} \cdot \lambda_{\max }^{\mathbf{M}_{S}} / 2$, and $\eta / 2=\zeta^{\dagger} \zeta$. Moreover, in order to get the pdf of $\eta$, the combination of random variables $\varepsilon_{n}, n=0,1, \cdots, N_{R}-1.5$ is necessary. Finally, by using of the definition of stochastic process the characteristic function $(\mathrm{CF})$ can be determined as

$$
\begin{aligned}
C F(\phi) & =E\left[\exp \left(\phi \cdot \Xi(S, y) / \log _{2} e\right)\right] \\
& =E\left[\log _{2}\left|\boldsymbol{I}_{\mathrm{t}}+\boldsymbol{Q}_{\mathrm{t}}\right|\right]
\end{aligned}
$$


The results of channel capacity for a 3-D M-MIMO beamforming system with MRC versus the mutual information can be easily obtained by means of the formula defined previously of CF. Let's consider an M-MIMO system with the STSK constellation modulation operating in 3-D channel environment in which has the AoA and AoD conditions. Normally, the performance evaluation of BER for the M-MIMO system deployment isn't consider both the parameters of AoA and DoA, because both the AoA and DoA are simply omitted in short distance transmission under indoor environment. Thus, in fact the vertical angle will become an important variable to affect the channel capacity of the M-MIMO beamforming system. It is known that the squared value of the intensity $z_{n}$ is a $\alpha-\lambda-\mu$ distribution as shown in (6). As the intensity $Z_{n}, n=0,1, \cdots, N_{R}-1$ are assumed as $\alpha-\lambda-\mu$ distributed which dominates the fading channel in dense environment. Moreover, the pdf of $S N R, \eta=\sum_{n=0}^{N_{R}-1} z_{n}$, conditioned on AoA and AoD can be obtained as [20]

$$
f\left(\eta \mid \phi_{e}, \theta\right)=\sum_{n=0}^{N_{R}-1} A_{E}\left(\phi_{e}, \theta\right) \cdot \sum_{k=1}^{m_{n}} \Delta_{N_{R}}\left(n, k, m_{u}, \sigma_{u}, l_{q}\right) \cdot f_{Z_{n}}\left(z, m_{n}, \sigma_{n}, \theta\right)
$$

where $f_{Z_{n}}\left(z, m_{n}, \sigma_{n}, \theta\right)$ has shown in (7), $A_{E}\left(\phi_{e}, \theta\right)$ involves the AoA and AoD components aforementioned in (1), $u=0, \cdots, N_{R}-1, q=1, \cdots, N_{R}-1$, and $\Delta_{N_{R}}\left(n, k, m_{j}, \sigma_{j}, l_{q}\right)$ is a recursive formula given as

$$
\begin{array}{r}
\Delta_{N_{R}}\left(n, m_{n}-k, m_{u}, \sigma_{u}, l_{q}\right)=\frac{1}{k} \sum_{\substack{j, u, q=0 \\
n \neq u}}^{N_{R}-1} \frac{m_{u}}{\sigma_{n}^{j}}\left(\frac{1}{\sigma_{n}}-\frac{1}{\sigma_{u}}\right)^{-j} \times \\
\Delta_{N_{R}}\left(n, m_{u}-k+j, m_{u}, \sigma_{u}, l_{q}\right)
\end{array}
$$

Assigning $D$ and $R$ present as the distance between the mobile user and the BS and the radius of the BS disk, respectively. As the 3-D channel shown in Fig. 2, the joint pdf of AoA and AoD can be rewritten a compact form as [20]

$$
f_{\theta_{n, m}, \phi_{n, m}}\left(\theta_{n, m}, \phi_{n, m}\right)=\left\{\begin{array}{c}
\frac{D}{2 \pi R} \frac{\left|\sin \left(\theta_{n, m}\right)\right|}{\sin ^{2}\left(\theta_{n, m}-\phi_{n, m}\right)}, \quad\left(\theta_{n, m}, \phi_{n, m}\right) \in \square, \\
0, \quad \text { otherwise }
\end{array}\right.
$$

where $\square$ denotes the region expressed as

$$
\square \in\left\{\left(\theta_{n, m}, \phi_{n, m}\right) \mid 0 \leq \frac{D \cdot \sin \left(\theta_{n, m}\right)}{\sin \left(\phi_{n, m}-\theta_{n, m}\right)} \leq R, \theta_{n, m} \neq \phi_{n, m}\right\}
$$

where $-\pi \leq \phi_{n, m} \leq \pi$, and $-\sin ^{-1}(R / D) \leq \theta_{n, m} \leq \sin ^{-1}(R / D)$ are the degree of the AoA and AoD, respectively, $n$ and $m$ indicate the different cluster numbers. The CF of spatial correlation channel of the M-MIMO system becomes 


$$
\begin{aligned}
C F(\phi) & =\sum_{i=0}^{N_{R}-1} A_{E}\left(\phi_{e}, \theta\right) \sum_{k=1}^{m_{i}} \Delta_{N_{R}}\left(i, k, m_{u}, \sigma_{u}, l_{q}\right) \times \\
& \frac{1}{\sigma_{i}^{m_{i}}\left(m_{i}-1\right) !} \Omega^{-m_{i}} \Gamma\left(m_{i}\right) \psi\left(m_{i}, m_{i}+1-\phi, \frac{1}{\Omega \sigma_{i}}\right)
\end{aligned}
$$

In order to obtain the result of CF shown in the previous equation, there is a part of integral required to be figured out. The equivalent formula $\int_{0}^{\infty} e^{-g x} x^{h-1}(1+a x)^{-v} d x=a^{-h} \Gamma(h) \psi(h, h+1-v, g / a)$ has been applied in the calculation [22], where $\psi(i, j ; k)=\int_{0}^{\infty} e^{-k t} t^{\alpha-1}(1+t)^{j-i-1} / \Gamma(i) d t$ is the confluent Hypergeometric function. By applying the well known formula of differentiation of confluent Hypergeometric function [23]. Once the CF is determined from (20), the channel capacity of a 3-D M-MIMO beamforming system can be easily obtained by the mutual information formula shown in (13). Now the performance evaluation of BER can be analyzed by using the MGF of $S N R$, a helpful theorem to obtain the closed form of (12). Then the closed form of exact BER formula can be derived for STSK modulation constellations. Now by means of the theorem of iteration partial derivative $J(y)=\Pi_{i=1}^{N} f_{i}(y)$, then the equation (12) can be computed as [23]

$$
u_{q, l}(x)=(-1)^{\left(u_{q}-l\right)} \sum_{\Theta} \prod_{j=1, j \neq q}^{N}\left(\begin{array}{c}
u_{j}-1+i_{j} \\
i_{j}
\end{array}\right)\left\{\left(c x \alpha_{j}\right)^{-1}-\left(c x \alpha_{q}\right)^{-1}\right\}^{-\left(u_{j}+i_{j}\right)}
$$

where $\Theta$ indicates the set of nonnegative integers $\left\{i_{1}, \cdots, i_{q-1}, i_{q+1}, \cdots, i_{N}\right\}$ which satisfies that $i_{1}+\cdots+i_{q-1}+i_{q+1}+\cdots+i_{N}=u_{q}-l$.

Suppose that STSK constellation is deployed to the proposed 3-D M-MIMO beamforming system. Besides, it is considered that the signal is located at the situation of $(1,0)$ when it is transmitted with the normal case. In order to determine the closed-form of BER for 3-D M-MIMO STSK constellation with beamforming system, firstly to calculate the pdf of the instantaneous SNR per bit is necessary. By taking the inverse Laplace transform of MGF in (8) and which becomes as

$$
f_{\gamma(x)}(\bar{\gamma}(x))=\frac{1}{A(x)}\left\{\sum_{q=1}^{N} \sum_{l=1}^{u_{q}} u_{q, l}(x) \times\left[\bar{\gamma}(x)^{l-1} /(l-1) !\right] \times \exp \left[-\left(\bar{\gamma}(x) / c x \alpha_{q}\right)\right]\right\}
$$

For concerning the right of previously equation, the pdf of the instantaneous SNR per bit results from the is then confirmed from the simulation shown in Fig. 4. Consequently, if one who wants to compute the performance of average BER, $P_{3-D M-M I M O}^{\mathrm{BER}}$, for a 3-D M-MIMO beamforming system with STSK constellation over the conditions with AoA and AoD. The first step has to obtain the marginal pdf from removing the joint parameters in (16) individually. Hence, it can be evaluated by substitute (16) and (18) into (8) and expressed as 


$$
\begin{aligned}
& P_{3-D M-M I M O}^{\mathrm{BER}}=\int_{0}^{\infty} \int_{\phi_{L}}^{\phi_{U}} \int_{\theta_{L}}^{\theta_{U}} f_{\theta_{n, m}, \phi_{n, m}}\left(\phi_{e}, \theta\right) \cdot f\left(\gamma_{s} \mid \phi_{e}, \theta\right) d \theta d \phi_{e} d \gamma_{s} \\
& =\frac{1}{A(x)}\left\{\sum_{q=1}^{N} \sum_{l=1}^{u_{q}} u_{q, l}(x) \cdot[1 /(l-1) !]\right\} \times \Im\left(\gamma_{s}, \rho, m_{j}, \sigma_{j}, l_{q}\right)
\end{aligned}
$$

where

$$
\begin{aligned}
& \mathfrak{T}\left(\gamma_{s}, \rho, m_{j}, \sigma_{j}, l_{q}\right) \\
& \square \int_{0}^{\infty} f_{\theta_{n, m}, \phi_{n, m}}\left(\phi_{e}, \theta\right) \cdot f\left(\gamma_{s} \mid \phi_{e}, \theta\right) \cdot \gamma_{s}(x)^{l-1} \times \exp \left[-\left(\gamma_{s}(x) / c x \alpha_{q}\right)\right] \cdot d \gamma_{s}
\end{aligned}
$$

Try to solve the closed-form for the last formula seems hardly completed, since there are many parameters involving in the integral process. Hence, the results from numerical discussion are going to be deployed in the next section. In Eq. (24) $\Delta_{N_{R}}(\cdot)$ has been defined in (12), and $A_{E}(\phi, \theta)$ is taken account into the calculation of $C_{3-D \text { M-мIмO }}$. The values are assigned as $\phi_{3 d B}=\theta_{3 \mathrm{~dB}}=65^{\circ}$ and then $A_{m}=30 \mathrm{~dB}$.

\section{RESULTS DISCUSSION}

The validation of the derived theoretical formulas for jpdf of AoA and DoA, and the performance evaluation of BER for a 3-D M-MIMO with STSK constellation modulation are numerically illustrated in this section. It is known that the best way to verify the correction for a formulation of probability function is to develop its' pdf. For validation to the accuracy of pdf provided in (6), the graphs for probability density function (pdf) with 2-dimension and 3-dimension are illustrated in Fig. 4 and Fig. 5, respectively. The former one is with the parameters $\alpha=1.75, \lambda=0.4$ and different $\mu$ values. On the other hand, the later one shown in Fig. 5 is demonstrating the pdf corresponding to 3 different elements, $\alpha, \lambda$, and $\mu$. The conditions are considered under the conditions without SNR and transmission and receiver numbers in current stage.

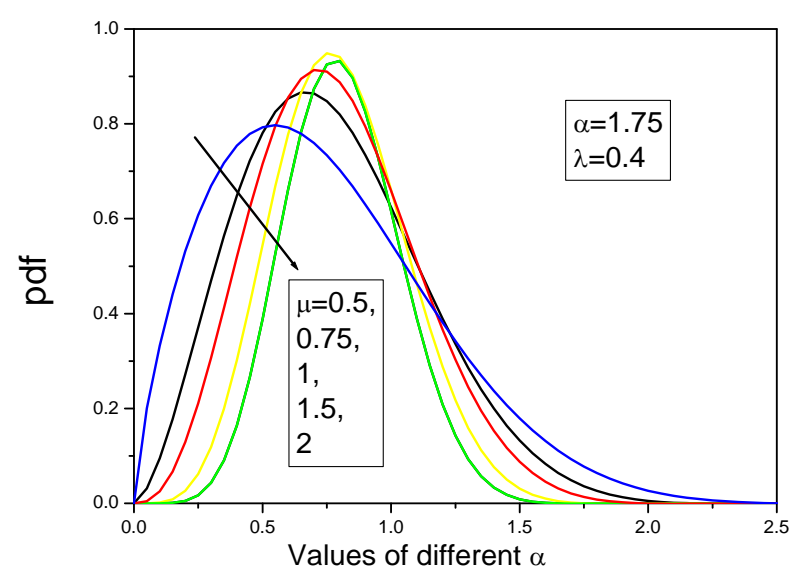

Fig. 4. The plots for the pdf of 3-D $\alpha-\lambda-\mu$ fading with $\alpha=1.75, \lambda=0.4$, and different $\mu$ values. 
The plots for the pdf of 3-D $\alpha-\lambda-\mu$ fading with $\alpha=1.75, \lambda=0.4$, and different $\mu$ values are shown in Fig. 4 , in which the obtained results are used to confirm the right formula derived in equation (22). Thus, the activity of the three parameters can be seized instantaneously. It is believed that the validation of the demonstrated graphics is accuracy when compared them with those originally shown in [23].

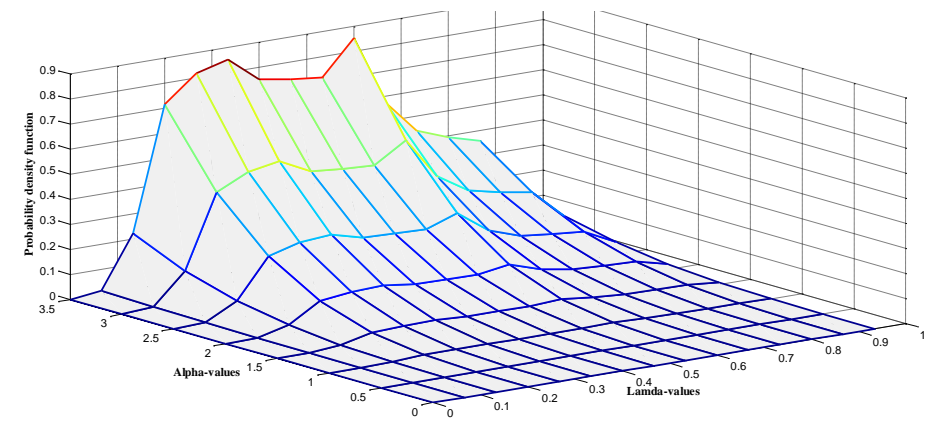

Fig. 5. The pdf of $\alpha-\lambda-\mu$ distribution with different $\alpha, \lambda$, and $\mu$ values.

Furthermore, the impact of correlation between azimuth and elevation angle parameters on the considered M-MIMO beamforming system is illustrated in Fig. 5.

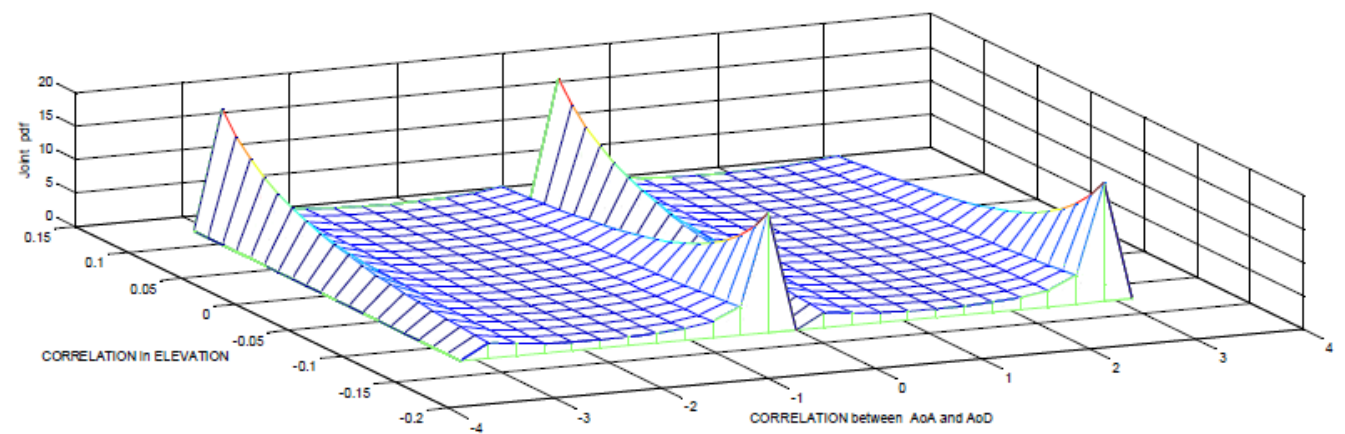

Fig. 6 The joint outcome for the correlation of AoA and AoD.

In Fig. 6 the mesh curves are illustrated corresponding to different correlation coefficient values. It is valuable to see that the correlation coefficient generally relates to the outcome of $d / \lambda$ which changes the size of AoA. Hence, the performance of channel capacity definitely becomes degradation when the correlation coefficient is promoted. Moreover, the phenomena of AoA parameter impact on the performance of BER for an M-MIMO beamforming system is illustrated in Fig. 5. The curves are corresponding to different $N_{T} \times N_{R}$ values. It is valuable to see the correlation coefficient that generally relates to the outcome of $d / \lambda$, and which changes the size of AoA. The analyzed results for accordance with that of 2-Dim channel model, the value of the elevation angle of the antenna boresight $\left(\theta_{T i t}\right)$ is 
assumed as fixed at $\pi / 2$. There are exactly $K$ antenna elements per antenna port with a pattern $A_{E}$ is given by $A_{E}\left(\phi_{e}, \theta\right)=G_{E, \max }\left\{-\left(A_{H}\left(\phi_{e}\right)+A_{V}(\theta)\right), A_{m}\right\}$, where $A_{m}=30 \mathrm{~dB}, G_{E, \max }=8 \mathrm{dBi} \quad A_{H}\left(\phi_{e}\right)=-\min \left[12\left(\phi_{e} / \phi_{3 \mathrm{~dB}}\right)^{2}, A_{m}\right], \phi_{3 \mathrm{~dB}}=65^{0}$, $A_{V}(\theta)=-\min \left[12\left(\theta-\theta_{\mathrm{tilt}} / \theta_{3 \mathrm{~dB}}\right)^{2}, \operatorname{SLAV}\right], \theta_{3 \mathrm{~dB}}=65^{0}$, and where $\operatorname{SLAV}=30 d B$ and the horizontal $3 d B$ is set as $\theta_{3 d B}=65^{0}$. In fact, the role which acted by an antenna element to complete the downtilt, ITU has provided the simplicity of global pattern for each port by a narrow beam and it is approximating to the form expressed as [3],

$$
\sqrt{\left[17 d B i-\min \left\{-\left(A_{H}\left(\phi_{e}\right)+A_{V}\left(\theta, \theta_{\text {titt }}\right)\right), 20 d B\right\}\right]_{l i n}}
$$

However, without loss the generality, the correlation coefficients are generated by the Gaussian correlation model of an equally spaced linear array, and which is with an arbitrary correlation coefficient. It is of interest to note that the correlation matrix followed by the linear array has a Toeplitz form constructed by correlation elements, $\rho_{i j}, i, j=0, \cdots, N_{R}-1,[20]$.

\section{CONCLUSION}

This work has analyzed the performance of BER and channel capacity for an M-MIMO system with STSK constellations scheme over 3-D correlated channel model which involves correlation phenomena between AoA and AoD. The calculation of MGF and CF are applied to avoid the hardness in determining the pdf of SNR, and the assumption with arbitrary correlation coefficient is arranged between the AoA and the AoD simultaneously. Moreover, both transmitter and receiver with different antenna numbers, $N_{T} \times N_{R}=2 \times 4$, are considered as the numerical analysis of an 3-D M-MIMO system with STSK constellations scheme for the verification purpose. Specifically, the evaluation results from the scenario considering the correlation value between AoD and AoA of 3D channel for an M-MIMO system have demonstrated better outperformance when the number of receiver antenna is much larger. Furthermore, it is worthy to note that the performance of BER and channel capacity for an M-MIMO radio system works over 3-D beamforming with STSK scheme are mainly dominated by correlation coefficient between the AoA and the AoD. Although, the discussion of performance for an M-MIMO system in 3-D fading channel applied to this article, to explore the performance calculation extends to consider the environment with other correlated channel, such as Weibull, and $\alpha-\lambda-\mu$ can be viewed as the other addressed issue in the future.

\section{References}

[1] P. Kyritsi and D. C. Cox, "Correlation Properties of MIMO Radio Channels for Indoor Scenarios," in Proc. of the Thirty-Fifth Conf. on Signals, Systems, and Computers, Asilomar, pp. 994-998, 2001. 
[2] X. Zhemin, S. Sana, and R. S. Blum, "Analysis of MIMO Systems with Receive Antenna Selection in Spatially Correlated Rayleigh Fading Channels," IEEE Trans. on Vehicular Technology, Vol. 58, no. 1, pp. 251-262, 2009.

[3] Nadeem, Qurrat-Ul-Ain, A. Kammoun, M. Debbah, Alouini Mohamed-Slim, “3D Massive MIMO Systems: Modeling and Performance Analysis," IEEE Transactions on Wireless Communications, Vol. 14, no. 12, pp. 6926-6939, Dec. 2015.

[4] Yi Wang; Chunguo Li; Yongming Huang; Dongming Wang; Tian Ban; Luxi Yang, "EnergyEfficient Optimization for Downlink Massive MIMO FDD Systems With Transmit Side Channel Correlation", IEEE Transactions on Vehicular Technology, Vol. 65, Issue: 9, pp. 72287243, Sep. 2016.

[5] Qing Guo; Guan Gui; Fei Li, "Block-partition Sparse Channel Estimation for Spatially Correlated Massive MIMO Systems", IEEE Conference in 2016 8th International Conference on Wireless Communications \& Signal Processing (WCSP), pp 1- 4, 2016.

[6] Qurrat-Ul-Ain Nadeem, Abla Kammoun; Merouane Debbah, Mohamed-Slim Alouini, "Performance Analysis of Compact FD-MIMO Antenna Arrays in a Correlated Environment", IEEE Access, Vol. Issue: 99, pp. 1-1, 2017,

[7] Young-Han Nam, Y. Li, J. C. Zhang, "3D Channel Models for Elevation Beamforming and FDMIMO in LTE-A and 5G," Signals, Systems and Computers, 2014 48th Asilomar Conference on , pp. 805-809, 2014.

[8] Shangbin Wu, Cheng-Xiang Wang, E. -H. M. Aggoune, M. M. Alwakeel, Yejun He, "A NonStationary 3-D Wideband Twin-Cluster Model for 5G Massive MIMO Channels," Selected Areas in Communications, IEEE Journal on, Vol. 32, Issue: 6, pp. 1207-1218, 2014.

[9] J. Charles, A. Furuskar, M. Frodigh, S. Jeux, A. Saadani, M. S. Hassan, A. Stidwell, J. Soder, B. Timus, "Refined Statistical Analysis of Evolution Approaches for Wireless Networks," Wireless Communications, IEEE Transactions on, Vol. 14, Issue: 5, pp. 2700-2710, 2015.

[10] Yuwei Ren; Xin Su; Can Qi; Yingmin Wang, "Channel Reconstruction for SVD-ZF Precoding in Massive 3D-MIMO Systems: Low-Complexity Algorithm”, 2016 IEEE 83rd Vehicular Technology Conference (VTC Spring), pp. 1-7, 2016.

[11] Yeqing Hu, Yi Hong, Jamie Evans, "Modelling Interference in High Altitude Platforms with 3D LoS Massive MIMO”, 2016 IEEE International Conference on Communications (ICC), pp. 1-6, 2016. 
[12] G. Sommerkorn, M. Kaske, C. Schneider, S. Hafner, R. Thoma, "Full 3D MIMO Channel Sounding and Characterization in an Urban Macro Cell, " General Assembly and Scientific Symposium (URSI GASS), 2014 XXXIth URSI, pp. 1-4, 2014.

[13] A. Kammoun, H. Khanfir, Z. Altman, M. Debbah, and M. Kamoun, "Preliminary Results on 3D Channel Modeling: From Theory to Standardization," IEEE Journal on Selected Areas in Commun., Vol. 32 , Issue: 6, pp. 1219-1229, 2014.

[14] S. Sugiura, "Dispersion Matrix Optimization for Space-Time Shift Keying," IEEE Communications Letters, Vol. 15, no. 11, NOV. 2011.

[15] C. SACCHI1, T. F. RAHMAN1, I. A. HEMADEH, and M. EL-HAJJAR, "Millimeter-Wave Transmission for Small-Cell Backhaul in Dense Urban Environment: a Solution Based on MIMOOFDM and Space-Time Shift Keying (STSK)," IEEE Journals \& Magazines, Vol. 5, pp. 4000 4017, 2017.

[16] Y. Wang, W. Zhang, O. Li, and P. Zhang, "Interference coordination in 3D MIMO-OFDMA networks," IEICE Trans. Communications, Vol. E97-B, no. 3, pp. 674-684, 2014.

[17]Qurrat-Ul-Ain Nadeem; Abla Kammoun; Mérouane Debbah; Mohamed-Slim Alouini, “3D Massive MIMO Systems: Modeling and Performance Analysis", IEEE Transactions on Wireless Communications, Vol. 14, Issue: 12, pp. 6926-6939, Dec. 2015.

[18] A. K. Papazafeiropoulos, S. A. Kotsopoulos, "The $\alpha-\lambda-\mu$ and $\alpha-\eta-\mu$ Small-Scale General Fading Distributions: A Unified Approach”, Wireless Pers. Commun., Vol. 57, pp. 735-751, 2011.

[19]Osamah S. Badarneh, Member, IEEE, and Mohammed S. Aloqlah, "Performance Analysis of Digital Communication Systems over $\alpha-\eta-\mu$ Fading Channels", IEEE Trans. on Vehicular Tech., pp. 79727981,Vol. 65, No. 10, Oct., 2016.

[20]A. Borhani and M. P"atzold, "Time-of-Arrival, Angle-of-Arrival, and Angle-of-Departure Statistics of a Novel Simplistic Disk Channel Model," Signal Processing and Communication Systems (ICSPCS), 2011 5th International Conference on, pp. 1-7, 2011.

[21] L. Liu, Y. Li, J. (Charlie) Zhang, "DoA Estimation and Achievable Rate Analysis for 3D Millimeter Wave Massive MIMO Systems", 2014 IEEE 15th International Workshop on Signal Processing Advances in Wireless Communications (SPAWC), pp. 6-10, 2014.

[22] I. S. Grodshteyn, I. M. Ryzhik, Table of Integrals, Series, and Products, 5th ed., CA: Academic Press, San Diego, 1994. 
J. Sustain. Wireless Syst.

Vol.01/ No. 01

Pages: 1-19

http://irojournals.com/irosws/

DOI: https://doi.org/10.36548/jsws.2019.1.001

[23] U. V. Amari and R. B. Misra, "Closed-form Expressions for Distribution of Sum of Exponential Random Variables,” IEEE Trans. on Reliab., Vol. 46, no. 4, pp. 519-522, Dec. 1997. 